\section{Detección e intervención oportuna en las caídas de los ancianos}

\section{Timely detection and intervention on falls among elderly adults}

\section{Señor Editor:}

Hemos leído con interés el artículo de Aliaga y colaboradores "Prevalencia de pacientes con alto riesgo de caídas en un servicio médico-quirúrgico de un hospital universitario" donde exponen la elevada frecuencia de pacientes con alto riesgo de sufrir una caída, describen sus características, siendo más frecuentes en aquellos casos con edad avanzada, déficit sensorial, alteración de la marcha y neurológica, historia previa de caídas y consumo de fármacos asociados a las caídas, y sobre todo destacan la baja implementación de medidas preventivas $^{1}$. Los autores comentan la posible pobre percepción del médico del riesgo de caídas entre las posibles causas de la baja implementación de intervenciones para prevenir las caídas ${ }^{1}$. En este sentido, nos gustaría compartir nuestra experiencia así como realizar una serie de comentarios sobre los resultados expuestos.

Se conoce que las caídas son una de las principales causas de lesiones, deterioro funcional, institucionalización y mortalidad en los adultos de edad avanzada ${ }^{2}$. Para ilustrar este aspecto, nos gustaría mostrar nuestros datos sobre las consecuencias inmediatas sufridas por los pacientes mayores atendidos por una caída en los servicios de urgencias españoles. El Registro FALL-ER, un estudio de cohortes prospectivo que incluyo 1.610 ancianos que habían sido atendidos por una caída en cinco servicios de urgencias españoles, documentó que $1.071(67,1 \%)$ casos tuvieron una lesión grave, 705 $(46,9 \%)$ miedo a volver a caerse, $298(19,4 \%)$ deterioro funcional agudo, y $24(1,6 \%)$ mortalidad durante el ingreso, y la frecuencia de estos eventos (lesión grave $\mathrm{p}=0,001$, miedo a volver a caerse $\mathrm{p}<0,001$, deterioro funcional agudo $p=0,116$, y mortalidad intrahospitalaria $\mathrm{p}=0,027$ ) se incrementó según avanzada la edad ${ }^{2}$. Y lo más importante, se halló que solo se realizaba prevención secundaria en tres de cada diez pacientes mayores previamente al alta de hospital ${ }^{3}$.

Estos datos ilustran la necesidad llevar a cabo estrategias de prevención de las caídas tanto por las consecuencias de las mismas como por la posible falta de percepción del riesgo por parte del equipo médico sobre su prevención primaria y secundaria. En este sentido, parece clave la identificación de los pacientes con alto riesgo de caída, especialmente entre aquellos ancianos que ya han sufrido una primera caída, ya que puede suponer grandes beneficios de cara a prevenir futuras consecuencias físicas, funcionales, psicológicas y económicas ligadas a las caídas ${ }^{4}$. Compartimos con los autores la reflexión sobre la necesidad de modificar el modelo de asistencia que ofrecemos al paciente anciano desde el primer contacto con el sistema sanitario. Además, pensamos que cualquier valoración médica realizada en el anciano debería ser multidimensional e incorporar de forma sistemática una valoración geriátrica que incluya la detección del riesgo de caídas. El desarrollo de herramientas de ayuda, informatizadas e integradas en la historia clínica del paciente, para la valoración y estratificación del riesgo de caídas, constituyen un paso fundamental para adaptar los recursos sanitarios disponibles a las necesidades del paciente anciano. Desde nuestro punto de vista, también sería necesario incorporar entre las recomendaciones de intervención para reducir el riesgo de caídas, especialmente entre aquellos con alto riesgo de sufrir una caída en el entorno sanitario, la mejora de las barreras arquitectónicas, la participación en programas de ejercicios diseñados para mejorar el equilibrio y la fuerza, más que la limitación de la movilidad, e intervenciones sobre problemas médicos específicos relacionados con las caídas.

Berenice Nayla Brizzi ${ }^{1}$, Montserrat Lázaro del Nogal $^{2,3}$, Francisco Javier Martín-Sánchez ${ }^{2,3,4}$ ${ }^{1}$ Servicio de Urgencias, Hospital Clínico San Carlos. Madrid, España.

${ }^{2}$ Unidad de Caídas, Servicio de Geriatría, Hospital Clínico San Carlos. Madrid, España.

${ }^{3}$ Facultad de Medicina, Universidad Complutense de Madrid. Madrid, España.

${ }^{4}$ Instituto de Investigación Sanitaria Hospital Clínico San Carlos (IdISSC). Madrid, España.

\section{Referencias}

1. Aliaga B, Molina N, Noguera M, Espinoza P, Sánchez S, Lara B, et al. Prevalencia de pacientes con alto riesgo de caídas en un servicio médico-quirúrgico de un hospital universitario. Rev Med Chile 2018; 146: 862-8.

2. Martín-Sánchez FJ, Brizzi BN, González del Castillo J, Fernández Pérez C, Canto Blázquez V, González Jiménez C, et al. Characteristics and outcomes of older adults presented to Spanish emergency departments after a fall. Eur Geriatr Med 2018; 9: 631-40.

3. Miró O, Brizzi BN, Aguilo S, Alemany X, Jacob J, Llorens P, et al. Perfil de los pacientes ancianos atendidos en urgencias por caídas (Registro FALL-ER): magnitud del problema y posibilidades de mejora en los servicios de urgencias hospitalarios. Emergencias 2018; 30: 231-40.

4. Vidan MT. Anciano con caída casual: signo de alarma general. Emergencias 2018; 30: 219-20.

5. Platts-Mills TF. El urgenciólogo y las caídas en personas de edad avanzada: ¿estamos preparados para la prevención primaria? Emergencias 2018; 30: 221-3.

Financiación: Este trabajo ha sido posible gracias a una beca de la Fundación MAPFRE (C.I. 14/371-E BS).

Correspondencia a:

Berenice Nayla Brizzi

Servicio de Urgencias. Hospital Clínico San Carlos.

Calle Profesor Martín-Lagos s/n, 28040 Madrid.

Telephone: (34) 91.330.37.50 / Fax: (34) 91.330.35.69

berenbrizzi@gmail.com 